\title{
Non-destructive residual stress investigations of natural polycrystalline diamonds
}

\author{
Andrew M. Venter ${ }^{1, a^{*}}$, Vladimir Luzin ${ }^{2, b}$, Marco A.G. Andreoli ${ }^{1,3, c}$, \\ Sandra Piazolo ${ }^{4, d}$ and Tshegofatso Moipolai ${ }^{1,5, e}$ \\ ${ }^{1}$ Research and Development Division, Necsa limited, Pretoria South Africa \\ ${ }^{2}$ Australian Nuclear Science and Technology Organisation, Australia \\ ${ }^{3}$ School of Geosciences, University of Johannesburg, South Africa \\ ${ }^{4}$ Department of Earth and Planetary Sciences, Macquarie University, Australia \\ ${ }^{5}$ Department of Physics, University of Johannesburg, South Africa \\ aandrew.venter@necsa.co.za, bvladimir.luzin@ansto.gov.au, 'marco.andreoli@necsa.co.za, \\ dsandra.piazolo@mq.edu.au, 'tshegofatsomoipolai0083@gmail.com
}

Keywords: Natural polycrystalline diamonds, non-destructive analysis, residual stress, X-ray and neutron diffraction.

\begin{abstract}
Three natural polycrystalline diamond samples have been investigated non-destructively in their raw as-discovered forms. The samples originate from different locations in the world and possibly have different mechanisms of formation. The study reveals that the stones are primarily composed of cubic diamond with varying amounts of impurities that emanate from their excessive porosities and entrapped environmental contamination, contributed by the areas in which they were formed and subsequently discovered. Residual stress analyses of the cubic diamond phase with Xray and neutron diffraction techniques revealed low stress values in the interior regions of the diamonds.
\end{abstract}

\section{Introduction}

Carbon occurs as a native element in several allotropes, the most familiar being graphite (very soft, hexagonal, black, as anisotropic sheets) and diamond (extremely hard, cubic, various colours, generally translucent). In geological formations, in addition to well-familiar single crystal diamonds, there are polycrystalline fine-grained aggregates (carbonado and related forms) and other rare forms [1]. Our research interest is in the non-destructive investigation of the residual stresses locked into polycrystalline diamond samples in their raw as-discovered form. Such results can extend the topical debate on their very controversial formation origin $[2,3]$. The typical, primary requirements for natural diamond formation are combinations of high pressures (HP) and high temperatures (HT). Possible mechanisms are [4]:

- Igneous crystallisation from C-rich kimberlite melts in the mantle [HT-HP].

- Solid state conversion from graphite due to subduction of oceanic/continental crust to mantle depth [HP-HT].

- Shock-metamorphism in meteorite impact processes [Ultra HT-PT].

- Presolar.

We report results from exploratory investigations that have been performed non-destructively on three polycrystalline diamond samples in their as-discovered forms using scanning electron microscopy (SEM) with energy dispersive spectrometry (EDS) analysis, X-ray diffraction (XRD), as well as stress analysis of the sub-surface and interior regions using X-ray and neutron diffraction (ND). No results on the residual stress mapping of the interior of a polycrystalline diamond stone were found in the literature making this investigation unique in this regard. 


\section{Samples}

The samples of this investigation comprised three polycrystalline diamonds discovered on the earth surface in soils/sediments and may have been formed from any of the mechanisms discussed [5]. One specimen among this set of samples is of the type commonly known as carbonado, a natural polycrystalline diamond of much disputed origin only found in alluvial deposits in the Central African Republic (CAR) and Brazil. These carbonandos are relatively porous masses of fine-grained interlocking micrometer-sized diamond grains that have hardness that exceeds that of diamond single-crystals. Although they do not have monetary value as gems, they are of industrial value owing to their superior abrasive qualities. The second sample also resembles a carbonado, being black, polycrystalline and extremely hard consisting of nanometer-scale diamonds. Unlike the true carbonados, this unusual stone named "Hypatia", was discovered in the area of southwest Egypt where an extreme surface heating event produced the Libyan Desert Glass 28.5 million years ago. Unlike true carbonados, Hypatia is non-porous and intensely fractured. A more detailed description of this stone has been provided by Kramers et al. [6] proposing that the Hypatia stone was a remnant of a cometary nucleus fragment that impacted after incorporating gases from the atmosphere based on noble gas isotopes and $\delta^{13} \mathrm{C}$ values. The third sample of this study originates from Northern Lesotho (NL), is light grey in colour and substantially larger than the other samples with a largest dimension of $30 \mathrm{~mm}$. The NL sample is from the Liqhobong kimberlitic pipes (the Satellite Pipe, to be distinguished from the Main pipe), and was provided by Andrew Burnie of Firestone Diamonds Ltd. to the ARC National Key Centre for Geochemical Evolution and Metallogeny of Continents (GEMOC) of the Department of Earth and Planetary Sciences at Macquarie University.

\section{Experimental methods and results}

With the samples being of significant scientific value, no surface preparation, apart from superficial surface cleaning to remove loose deposits, was performed. Characterisation investigations of the cabonado and Hypatia samples included SEM and EDS for elemental composition determination and XRD for mineral phase identification. Results are summarised in Table 1.

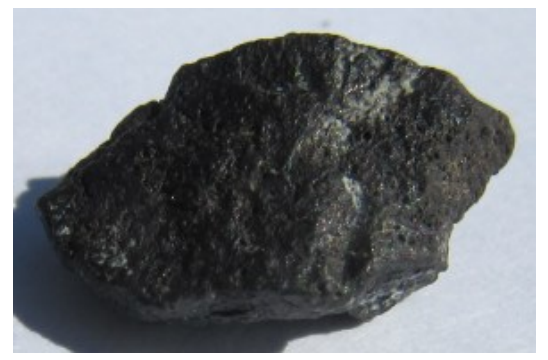

(a)

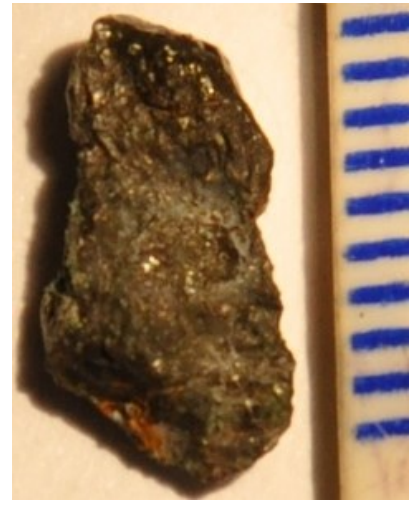

(b)

Figure 1: Photographs of: (a) the carbonado sample originating from Brazil $\left(10 \times 9 \times 8 \mathrm{~mm}^{3}\right.$ in size $)$ and (b) the Hypatia sample emanating from North Africa $\left(10 \times 5 \times 2 \mathrm{~mm}^{3}\right.$ in size). 
Table 1: Summary of the Brazilian and Hypatia stone compositions. Stress values were determined with XRD at corresponding positions on the samples.

\begin{tabular}{|c|c|c|c|c|c|c|c|}
\hline \multirow{2}{*}{$\begin{array}{l}\text { Sample } \\
\text { description }\end{array}$} & \multicolumn{2}{|c|}{ Elemental content (EDS) } & \multicolumn{2}{|c|}{ Mineral composition (XRD) } & \multicolumn{2}{|c|}{$\begin{array}{l}\text { Phase content } \\
\text { (XRD) [wt.\%] }\end{array}$} & \multirow{2}{*}{$\begin{array}{c}\text { Residual } \\
\text { stress } \\
\text { [MPa] } \\
\end{array}$} \\
\hline & Major & Minor & Major & Minor & Diamond & Impurities & \\
\hline Brazilian & $\mathrm{C}(84 \%)$ & $\begin{array}{c}\mathrm{O}(6 \%) \\
\mathrm{Ca}(5 \%)\end{array}$ & $\begin{array}{c}\text { Cubic } \\
\text { diamond }\end{array}$ & $\begin{array}{c}\mathrm{SiO}_{2} \\
\mathrm{CaCo}_{3}\end{array}$ & $98 \%$ & $2 \%$ & $\begin{array}{l}-25 \pm 40 \\
130 \pm 40\end{array}$ \\
\hline Hypatia & $\mathrm{C}(50 \%)$ & $\mathrm{O}(43 \%)$ & $\begin{array}{c}\text { Cubic } \\
\text { diamond }\end{array}$ & $\begin{array}{c}\text { Oxide and } \\
\text { hydroxide } \\
\text { dominance: } \\
\mathrm{SiO}_{2} \text {. } \\
\mathrm{Kaolinite} \\
(\mathrm{Al})_{2}(\mathrm{Si})_{2} \mathrm{O}_{5} \\
(\mathrm{OH})_{4}\end{array}$ & $76 \%$ & $24 \%$ & $\begin{array}{l}-90 \pm 100 \\
110 \pm 100\end{array}$ \\
\hline
\end{tabular}

Carbon is the dominant element in both these samples, but with a significant oxygen content present in the Hypatia sample (in agreement with the results from [6]). The latter could not unambiguously be linked to specific metal oxides. The presence of silicon in the EDS spectra is ascribed to surface contamination with $\mathrm{SiO}_{2}$ (quartz in sand) and clays (e.g. kaolinite). The diffraction analysis revealed the samples to be fine grained and the crystalline carbon being present as cubic diamond. No lonsdaleite, the high-pressure carbon polymorph characteristic of meteorite impact diamond [7,8] was unambiguously identified in the Hypatia sample. Both samples show the presence of impurity phases to different extents. Using a Rietveld refinement approach, it was estimated that the Brazilian and Hypatia samples consist of $98 \mathrm{wt} . \%$ and $76 \mathrm{wt} . \%$ cubic diamond versus 2 wt.\% and 24 wt.\% combined impurities respectively. In addition, by comparing the diffraction patterns of these two carbonado samples, significant peak broadening is evident in Hypatia as shown by Fig. 2. This is indicative of the presence of nano-crystalline diamond in addition to substantial mechanical deformation with extensive accumulation of dislocations and defects.

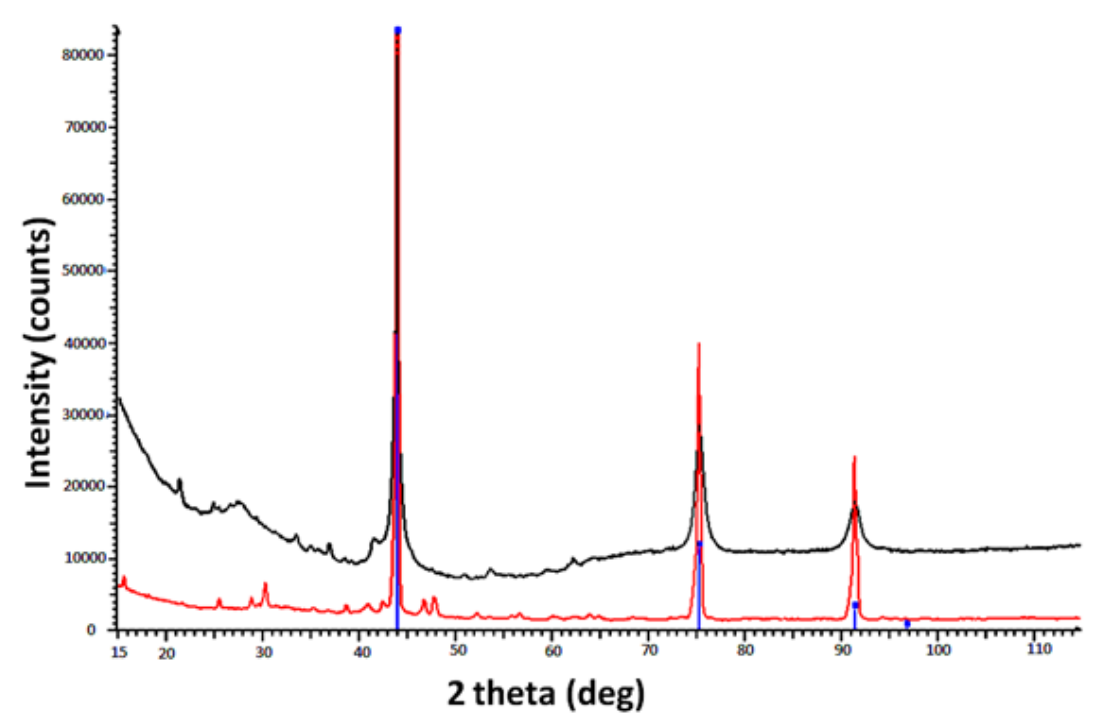

Figure 2: Overlaid X-ray diffraction patterns of the Brazilian (red) and Hypatia (black) samples. Both samples have cubic diamond as the major phase (indicated by the blue stick pattern) with quartz and calcite as minority phases without clear dominance. 
The next phase of the investigation was the determination of residual stresses present in the bulk polycrystalline diamonds. The Brazilian and Hypatia samples were investigated with XRD using a Bruker D8 Discover micro-diffraction instrument equipped with a $0.8 \mathrm{~mm}$ diameter beam and $\mathrm{Cu}$ radiation rendering penetration depths in the order of $250 \mu \mathrm{m}$ into the diamond phase. Measurements were done using the $\sin ^{2} \psi$ technique based on a linear dependence existing between the strain and $\sin ^{2} \psi$, with $\psi$ being the tilt angle from the surface normal. The (311) reflection provided the highest diffracted intensity and most favourable grain statistics owing to its high multiplicity factor, $M=24$. The irregular sample surfaces though caused a breakdown in the linear dependence at $\sin ^{2} \psi>0.6$. Data analysis was subsequently limited to the linear region only. Stress values determined at a number of locations on the sample surfaces are summarized in Table 1.

Since the X-ray results are susceptible to surface effects with associated questionable accuracy, as a next phase of the project, thermal neutron strain scanning [9] was employed for the nondestructive investigation of the interior regions of the bulk diamonds by exploiting the superior neutron scattering properties of carbon and high penetration of thermal neutrons into the material. This in conjunction with the gauge volume size employed (larger with the X-ray experiment) led to the probing of significantly more grains [9]. In this unique approach, the Kowari neutron strain scanner [10] was used to investigate the central interior regions of the Hypatia stone using a gauge volume of $1 \times 1 \times 1 \mathrm{~mm}^{3}$ and a wavelength of $1.52 \AA$ that placed the diamond (311) reflection at the optimal $90^{\circ}$ gauge volume definition geometry. The measurements were done on the center line of the sample mid-thickness along the largest dimension of the sample. The tri-axial stress results are shown in Fig. 3. The statistical uncertainties in the neutron counts were relatively large due to the broad Bragg peaks contributing to stress uncertainties of approximately $80 \mathrm{MPa}$. Stress variations are ascribed to statistical variations rather than a real stress distribution. Notwithstanding the stress variations being larger than the error bars, no apparent trend could be deduced. Assuming no stress, the spread in the experimental data points is approximately $200 \mathrm{MPa}$. Since this cannot be explained in terms of the counting or grain statistics as the grain size is extremely small, possible causes are instead associated with local variations in the microstructure (e.g. defects, macroscopic voids and other phase inclusions) and localized microstresses (interaction with other phases and stress concentrations around defects).

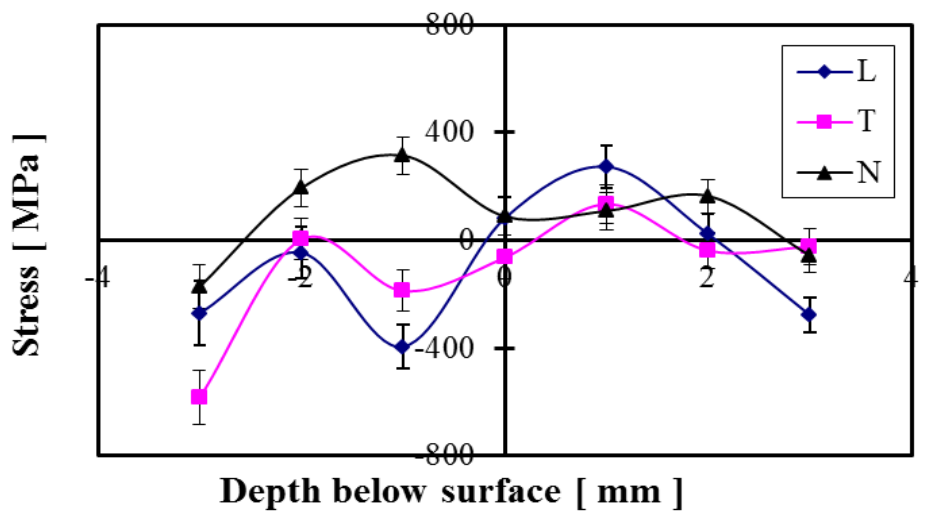

Figure 3: Residual stress measured along three orthogonal directions in the Hypatia sample. The error bars shown were determined from the counting statistics and peak fitting accuracy which are in the order of $\pm 80 \mathrm{MPa}$.

Stress contour maps of the interior of the Hypatia sample were not possible due to the smallness of the sample. To gain a clearer understanding of the stresses in the interior of polycrystalline diamonds, a large sample originating from Northern Lesotho (NL) was available that enabled comprehensive 2D area stress mapping of the cross-section. This polycrystalline sample comprised a conglomerate of many visible small single crystallites that were large with respect to the $1 \times 1 \times 1$ $\mathrm{mm}^{3}$ neutron gauge volume. To overcome the associated grain statistics limitation, a multiple direction strain measurement approach was adopted to resolve the full stress tensor. Experimentally 
this required stringent sample positional accuracy with reference to the gauge volume (achieved with the use of high-precision omega and xyz sample stages). The measurement protocol comprised:

- Measurements at tilts along two perpendicular planes (that can be called $\psi$ - and $\varphi$-tilts) in $5^{\circ}$ increments with a total of 72 directions per measurement position to enable reconstruction of all five stress components, i.e. principal and shear stresses.

$\circ$ At the selected $(\varphi, \psi)$ directions, measurements were taken to render interatomic spacing accuracies better than $7 \times 10^{-5}$, resulting in $\sim 25 \mathrm{MPa}$ stress uncertainty due to neutron counting statistics, together with statistical scatter due to partial illumination effects from large single grains (grain statistics) [11].

o Overall, measurements were done in the rectangular cross-section, matrix of $11 \times 7$ with $2.5 \mathrm{~mm}$ separations that amounted to 77 measurement locations in the largest available cross-section.

$\circ$ For each location, the measured strains in these directions were fitted with a 6-component stress tensor using $\chi^{2}$-minimization and assuming a constant $\mathrm{d}_{0}$-value for the whole sample.

Figure 4 shows a photograph of the NL sample and the results of the tri-axial residual stress analyses. In all cases the overall stress values lie between -300 and $300 \mathrm{MPa}$. This needs to be viewed in relation to the large grain statistic contribution. A statistical analysis of the data predicts statistical errors 4-5 times larger than the experimental error bars shown, so the total error bar would be $\pm(100-150) \mathrm{MPa}$. Most likely these can be attributed to statistical uncertainties other than neutron counting or grain statistics. Assuming this accuracy, it is concluded that there is no significant difference between the stress components and thus no obvious evidence of stress distribution/pattern.

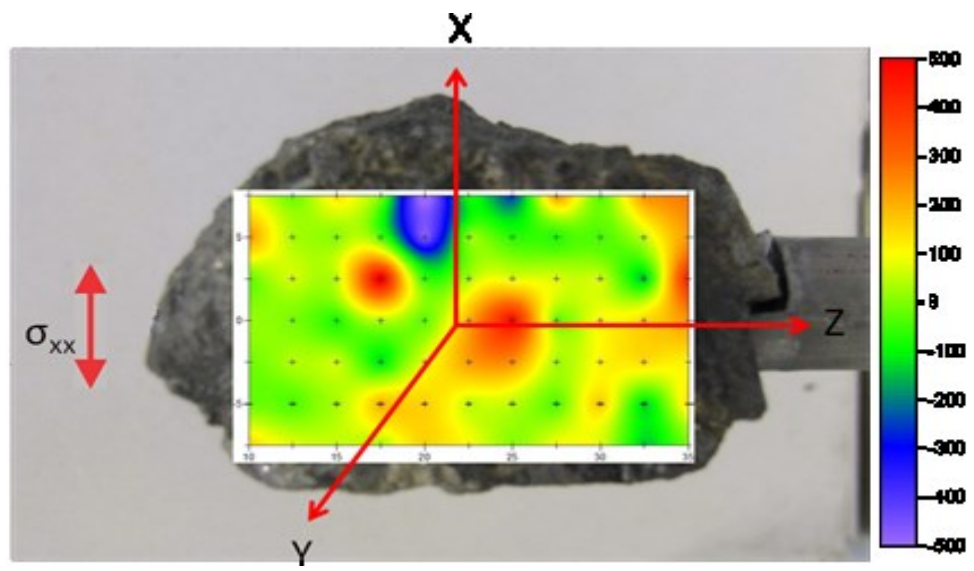

(a)

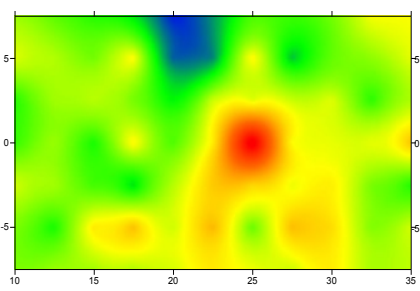

(b)

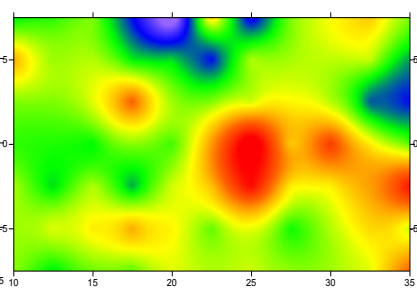

(c)

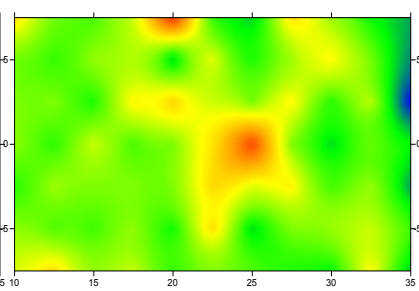

(d)

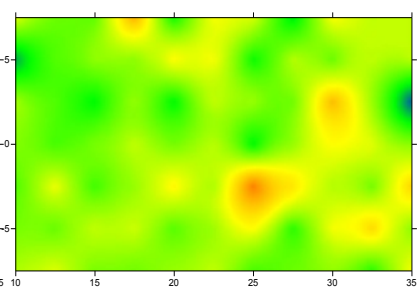

(e)

Figure 4: (a) Residual stress of the assumed $\sigma_{x x}$ component determined along a sheet in the centre of the Northern Lesotho polycrystalline diamond shown overlaid onto the sample shape and size (30 x $20 \times 15 \mathrm{~mm}^{3}$ in size). The additional contour plots display (b) $\sigma_{\mathrm{yy}}$; (c) $\sigma_{\mathrm{zz}}$; (d) $\sigma_{\mathrm{xy}}$ and (e) $\sigma_{\mathrm{xz}}$ stress components.

\section{Discussion}

Agreement exists between the stresses determined at $250 \mu \mathrm{m}$ depths (XRD) and the sample mid-thicknesses (ND) for the Hypatia sample: Within the accuracy rendered by the grain statistics, i.e. $\pm 150 \mathrm{MPa}$, it is concluded that no global residual stress exists in the interior regions, or subsurface regions of the sample. The comprehensive ND investigation of the NL samples reveals 
similar results. This indicates that the samples had formed in relatively uniform temperature and pressure environmental conditions. However, some local variations of stress in the range of \pm 300 $\mathrm{MPa}$ were observed experimentally, whilst the experimental accuracies were much smaller, $25 \mathrm{MPa}$ for NL and $80 \mathrm{MPa}$ for Hypatia. These variations appear to be statistical in nature and attributed to inhomogeneity in the morphology and stress concentration due to structural defects [12], microstress due to the presence of other phases, and variation of $\mathrm{d}_{0}$ due to accumulation of dislocations that have been reported in diamond samples [13].

The localization of stresses observed in our study is corroborated by other experimental evidences. Han et al. [14] reported stress results of a plane-wave based ab initio pseudo potential technique (computational) in a single-crystalline sample and indicated that the stress was dependent on the atomic surface termination species. They predict -35 GPa for nitrogen and $26 \mathrm{GPa}$ in the case of boron at depths of a few atomic layers, i.e. nanometer range. Kagi et al. [15] reported a tensile stress of $0.49 \mathrm{GPa}$ using Raman Spectroscopy at a sub-surface depth of $10 \mu \mathrm{m}$ on a polished Carbonado sample from the CAR. Thus based on our XRD and ND results taken at $250 \mu \mathrm{m}$ and millimetre depths respectively, and in relation to the published results, indications are that a steep stress gradient may exist within the $0.2 \mathrm{~mm}$ from the surface, but effectively low stress values exist in the sample interiors.

\section{Acknowledgements}

The authors acknowledge support from Necsa Ltd., the use of their SEM and XRD laboratories, as well as ANSTO for the beam time awarded on the KOWARI instrument under proposal number 2705. Mr. Van der Merwe and Mr. Ntsoane are thanked for their assistance with the SEM and XRD investigations, as well as Dr. Andreoli and Dr. Piazolo for making the samples available for the investigations.

\section{References}

[1] E.I. Erlich and W.D. Hausel, Diamond Deposits, Society for Mining, Metallurgy and Exploration, Inc. USA, 2002 ISBN 0-87335-213-0.

[2] Y.A. Litvin, Russian Geology and Geophysics 50 (2009)1188-1200.

[3] K. Shibata and H. Kamioka Mineralogical Magazine 57 (1993) 607-611.

[4] G. Kletetschka, P.T. Taylor and P.J. Wasilewski, H.G.M. Hill, Earth and Planetary Science Letters 181 (2000) 279-290.

[5] M. Ozima, S. Zashu, K. Tomura and Y. Matsuhisa, Nature 351 (1991) 472-474.

[6] J.D. Kramers, M.A.G. Andreoli, M. Atanasova, G.A. Belyanin, D.L. Block, C.B. Franklyn,C. Harris, M. Lekgoathi, C.S. Montross,T.P. Ntsoane, V. Pischedda, P. Segonyane, K.S. Viljoen and J.E. Westraadt, Earth and Planetary Science Letters 382 (2013) 21-31.

[7] R. Z. Khaliullin, H. Eshet, T. D. Kühne, J. Behler and M. Parrinello, Nature Materials 10 (2011) 693-697.

[8] C. Frondel and U.B. Marvin, Nature 214 (5088) (1967) 587-589. Bibcode:1967Natur.214..587F. doi:10.1038/214587a0

[9] P. Withers in: M.E. Fitzpatric, A. Lodini (Eds.), Analysis of Residual Stress by Diffraction Using Neutron and Synchrotron Radiation, Taylor and Francis, London, 2003, p. 170.

[10] O. Kirstein, U. Garbe and V. Luzin, Mater. Sci. Forum 652 (2010) 86.

[11] A. Steuwer, J. R. Santisteban, M. Turski, P. J. Withers and T. Buslaps, J. Appl. Cryst. 37 (2004) 883-889.

[12] V.A. Petrovsky, A.A. Shiryaev, V.P. Lyutoev, A.E. Sukharev and M. Martins, Eur. J. Mineral. 22 (2010) 35-47.

[13] D. Howell, S. Piazolo, D.P. Dobson, I.G. Wood, A.P. Jones, N. Walte, D.J. Frost, D. Fisher, and W.L. Griffin, Diamond Relat. Mater. 30 (2012) 20-30.

[14] S. Han and J. Ihm, Physical Review Letters 80(5) (1998) 995-998.

[15] H. Kagi and S. Fakura, Eur. J. Mineral. 20 (2008) 387-393. 